\title{
Iodine Molecules Differential Absorption Cross section Lidar Studies
}

\author{
V.E. Privalov, V.G. Shemanin ${ }^{1}$, E.I.Voronina ${ }^{1}$ \\ St. Petersburg State Polytechnic University, Politechnicheskaya, 29, 195257, Saint - Petersburg, \\ Russia, vaevpriv@yandex.ru \\ ${ }^{1}$ Kuban State Technological University, Novorossiysk Polytechnic Institute, K. Marx, 20, 353900, Novorossiysk, \\ Russia, vshemanin@nbkstu.org.ru
}

Lidar monitoring of the gaseous molecules in atmosphere can be used for concentration measurements of the toxic pollutants in air of the urban and industrial regions. Such a concentration study in the multi-components gaseous mixture by lidar technique is a complex problem in atmospheric pollution monitoring. The differential absorption lidar (DA-lidar) is the most preferable for the detection of iodine molecules at very low concentration levels. The iodine molecules absorption cross section in the laboratory lidar studies and lidar equation simulation in this lidar variant corresponds with earlier data.

Key words: iodine molecules, concentration, lidar, differential absorption cross section

\section{INTRODUCTION}

$\mathrm{L}$ IDAR SYSTEMS for gaseous molecule concentration measurements in multi - component gas mixture monitoring have been realized by any spectroscopic variants determined by the studied molecules $[1-4]$. Molecular iodine concentrations measurements in technological gases and atmosphere upper AES or other radiochemical enterprises are of interest because its heavy isotopes ${ }^{131} \mathrm{I}_{2}$ can serve as indicators of air quality [4]. The lidar equation computer simulation has been performed earlier in $[2,5,7]$ for all lidars and it has been concluded that differential absorption lidar (DA-lidar) is the most preferable for the iodine molecules detection at low concentration levels. The goal of this paper is experimental studies of molecular iodine DA-lidar differential absorption coefficient versus iodine concentration dependences and DA-lidar equation computer simulation.

\section{EXPERIMENTAL SETUP}

The laboratory experimental setup is shown in Fig.1. YAG: Nd Q-switching laser (1) beam with pulse duration of $10 \mathrm{~ns}$ and pulse energy about $25 \mathrm{~mJ}$ at wavelength $532 \mathrm{~nm}$ and $40 \mathrm{~mJ}$ at $1064 \mathrm{~nm}$ of laboratory on-axial lidar with ranging distance up to $8 \mathrm{~m}$ was directed into special Brewster windows - ended by a vacuum cuvette (4) with $\mathrm{I}_{2}$ molecules. The $532 \mathrm{~nm}$ wavelength YAG: Nd laser (1) radiation intensity of laboratory lidar passing through cuvette (4), was directed to FD-24K type photodiode (6) with interference filter (7) at this wavelength. The $1064 \mathrm{~nm}$ wavelength laser (1) radiation intensity passing through vacuum cuvette (4) was directed by the dichromatic mirror (5) to the same type photodiode (9) with interference filter (8) too. It served as reference beam in the differential measuring scheme. The portions of both wavelengths of the laser (1) beams were turned by two glass plates (2) and (3) to the same type of photodiode (12) and (11) with two interference filters (13) and (14). The $I_{2}$ concentration in cuvette (4) was varied by cuvette appendix heating in the water heater (10) from 25 to $85^{\circ} \mathrm{C}$. Four photodiode pulse outputs of the same type were recorded by memory oscilloscope or imported into a special PC-controlled measuring system as the DA coefficient $X$ that can be determined from DA-lidar equation [2, 8] and is equal to $X=\sigma_{0} N \Delta R$, where the absorption cross section, iodine molecules concentration and absorbing layer thickness multiplier is in the right part. The cuvette (4) thickness from window to window is about $10 \mathrm{~cm}$. The iodine molecules concentration level $N$ was varied from $710^{14} \mathrm{~cm}^{-3}$ up to 3.4 $10^{16} \mathrm{~cm}^{-3}$ in our case. The sensing distance range of $1 \ldots 8$ $\mathrm{m}$ has been used in this study.

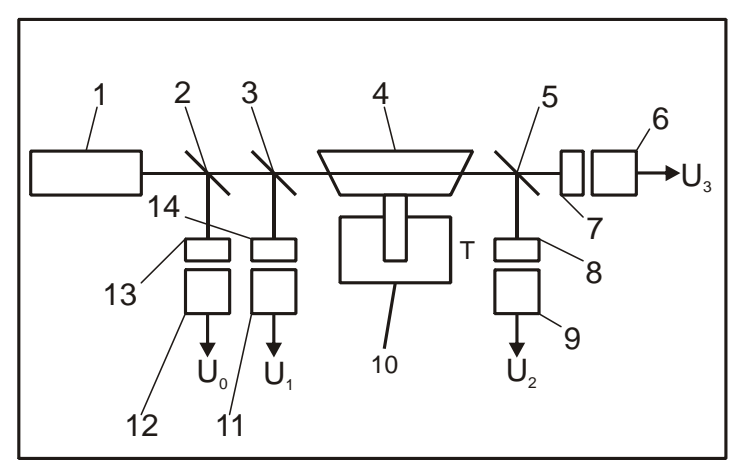

Fig.1. Experimental lidar layout

\section{DA - LIDAR STUDY RESULTS}

The $532 \mathrm{~nm}$ wavelength YAG: Nd laser radiation intensity of laboratory DAL lidar [2,6] that passed through cuvette was decreased due to iodine molecules absorption [3,9] and has been directed to the FD-24K type photodiode with interference filter at this wavelength. The $1064 \mathrm{~nm}$ wavelength laser $\mathrm{FH}$ radiation intensity that passed through vacuum cuvette with $I_{2}$ has been recorded too. It served as reference beam in the differential measuring scheme. Four 
photodiode pulse outputs of the same type were recorded by memory oscilloscope. These photodiode output measuring results at $4 \mathrm{~m}$ distance, for example $U_{0}$ and $U_{1}$ - the 1064 and $532 \mathrm{~nm}$ wavelength signals before cuvette, but $U_{2}$ and $U_{3}$ - the same wavelength signals after passing through the cuvette in Fig.1., were converted into pulse energy values. The calibration studies were at first fulfilled for the photodiode receiving module transmission coefficient measuring. This transmission coefficient value $K_{f}=$ $4.5 \pm 0.5 \mathrm{~mJ} / \mathrm{V}$ was established by photodiode output amplifier coefficient change. These results allowed us to convert the output amplitude to pulse energy $E$ in Table 1 . The DA coefficient $X$ values are shown in the last column of Table 1 . The average measurement ratio error was equal to $8.5 \%$ in our case. The measuring results at the sensing distance range from 1 to $8 \mathrm{~m}$ were coincided with our measurement accuracy. The plot of DA coefficient $X$ (in arbitrary units) versus iodine molecules concentration $N$ in $10^{15} \mathrm{~cm}^{-3}$ is exhibited in Fig.2. The iodine molecules absorption cross section at $532 \mathrm{~nm}$ wavelength was calculated as in [1]. It equals $\sigma_{0}=(1.88 \pm 0.37) 10^{-18} \mathrm{~cm}^{2}$ in satisfactory confidence with data [3] $-4.610^{-18} \mathrm{~cm}^{2}$ at 590 nm wavelength.

Table 1. The measured 1064 and $532 \mathrm{~nm}$ wavelengths DA pulse energy values, calculated iodine molecules concentration and DA coefficient values.

\begin{tabular}{|c|c|c|c|c|c|c|}
\hline $\begin{array}{c}N 10^{-15}, \\
\mathrm{~cm}^{-3}\end{array}$ & $\begin{array}{l}E_{0}, \\
\mathrm{~mJ}\end{array}$ & $\begin{array}{l}E_{l}, \\
\mathrm{~mJ}\end{array}$ & $\begin{array}{l}E_{2}, \\
\mathrm{~mJ}\end{array}$ & $\begin{array}{l}E_{3}, \\
\mathrm{~mJ}\end{array}$ & $\begin{array}{r}\Delta E_{0}, \\
\mathrm{~mJ}\end{array}$ & $X$ \\
\hline 7.42 & \multirow{6}{*}{45} & \multirow{6}{*}{20} & 23 & 11.7 & \multirow{6}{*}{0.9} & 0.51 \\
\hline 14.8 & & & 23 & 13 & & 0.59 \\
\hline 18.2 & & & 22 & 13.8 & & 0.63 \\
\hline 23.9 & & & 23 & 16 & & 0.69 \\
\hline 27.5 & & & 23 & 17 & & 0.75 \\
\hline 32.4 & & & 22 & 18 & & 0.82 \\
\hline
\end{tabular}

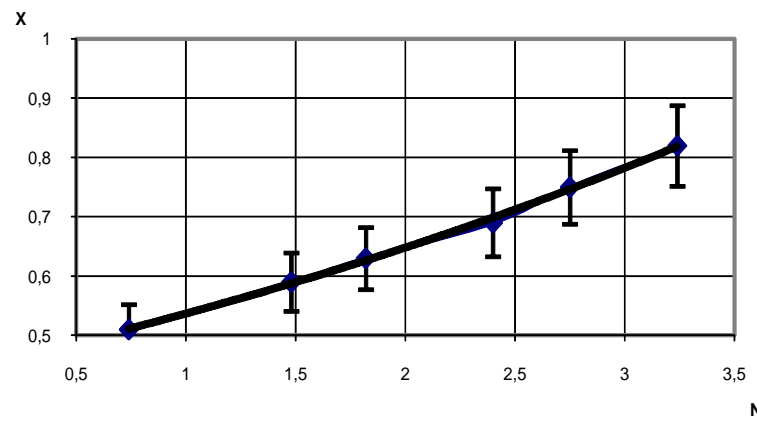

Fig.2. Plot of the DA coefficient $\mathrm{X}$ values vs. iodine molecules concentration $N$ in $10^{15} \mathrm{~cm}^{-3}$ at sensing distance of $4 \mathrm{~m}$.

\section{DA-LIDAR EQUATION SIMULATION}

The molecular iodine DA-lidar differential absorption coefficient versus iodine concentration dependences experimental studies can be confirmed by the DA-lidar equation computer simulation. The resonance absorption in the visible and infrared region of the spectrum has the interaction maximal cross section [3], [8] from all of the spectroscopic effects. The molecule low level concentration at the large ranging distance measurement possibilities was determined by this absorption. As it has been shown earlier, DA-lidar has two laser beams with different wavelengths, the first wavelength lies in the molecule absorption line maximum and the second wavelength lies out of this line and weakening of these beams was determined by the iodine molecules in the atmosphere. The studied molecule concentration distribution in atmosphere data were calculated by the recorded DA-lidar signal ratio at these two wavelengths in the narrow spectral interval $[1,3]$.

DA-lidar equation [2, 3, 8] simulation has been fulfilled for $\mathrm{I}_{2}$ molecules sensing possibilities estimation and molecules concentration and ranging distance ranges determination. The iodine molecules spectrum in the visible and infrared region consists of the electronic, vibration and rotation bands transitions from the basic state $X^{l} \Sigma^{+}{ }_{0 q}$ to the state $A^{3} \Pi_{l u}$, or $B^{3} \Pi^{+}{ }_{0 u}$ and ${ }^{1} \Pi_{u}$ [9] and the bands of such a transition from $X$ to the first electronic state $B$, lie in the range from 499 up to $670 \mathrm{~nm}$. The $532 \mathrm{~nm}$ wavelength YAG: Nd laser lies in this absorption band [1,9] and such an experimental situation is of interest for DA-lidar equation computer simulation.

The lidar equation for the elastic back scattering was rewritten by $[3,8]$ in the form

$$
P(\lambda, R)=P_{L}\left(\lambda_{L}\right) K_{l} A_{0} T^{2}\left(\lambda_{L}, R\right) \rho / R^{2}
$$

where $P(\lambda, R)$ - the back scattering signal power at the photodetector at the wavelength $\lambda_{L}$ from the distance $R ; P_{L}$ $\left(\lambda_{L}\right)$ - the peak power of the laser radiation and its wavelength $\lambda_{L} ; K_{1}$ - lidar constant; $A_{0}-$ the receiving telescope square; $T\left(\lambda_{L}, R\right)$ - the atmospheric transmittance at the wavelength $\lambda_{L} ; \rho$ - the topographic target reflection coefficient. All of the information about iodine molecules concentration is in the multiplier $T\left(\lambda_{L}, R\right)$, meaning that the extinction coefficient $k\left(\lambda_{L}, R\right)$ can be exhibited in the general case as in $[3,8]$ in the equation

$$
k\left(\lambda_{L}, R\right)=k_{A}\left(\lambda_{L}, R\right)+N_{a}(R) \sigma_{0}\left(\lambda_{L}\right)
$$

where the first adding is the atmospheric extinction coefficient at the laser radiation wavelength without the iodine molecules and the second one is the $I_{2}$ molecules concentration and the resonance absorption cross section multiplication of these molecules.

The next step due to the differential absorption method [8] is dividing the two lidar equations (1) for the two laser radiation wavelengths $\lambda_{0}$ and $\lambda_{1}$, where the second laser radiation wavelength is out of the $\mathrm{I}_{2}$ absorption band, by one another. The dividing result is the differential absorption equation for the general case in the next form

$$
\begin{aligned}
& \frac{P\left(\lambda_{0}, R\right)}{P\left(\lambda_{1}, R\right)}=\frac{P_{0} K_{10} \rho_{0}}{P_{1} K_{11} \rho_{1}} \exp \left\{-2 \int_{0}^{R}\left[k\left(\lambda_{0}, R\right)-\right.\right. \\
& \left.\left.-k\left(\lambda_{1}, R\right)\right] d R\right\}
\end{aligned}
$$


The parameters in (3) in our experimental case are the next. The two laser radiation wavelengths are $\lambda_{0}=532 \mathrm{~nm}$ and $\lambda_{1}=1064 \mathrm{~nm}$ as in the differential absorption method. The $1064 \mathrm{~nm}$ laser radiation wavelength is out of the $\mathrm{I}_{2}$ absorption band but it lies in the atmospheric transparency region [10]. The lidar receiving telescope square $A_{2}=0.011$ $\mathrm{m}^{2}$, lidar constant $K_{2}=0.4$ at the $1064 \mathrm{~nm}$ wavelength [6] and iodine molecules absorption cross section as it has been derived from our experiments $\sigma_{0}=1.8810^{-18} \mathrm{~cm}^{2}$. The laser radiation peak power values are equal to $P_{L}=10$ and $100 \mathrm{~kW}$ and the two wavelengths' laser radiation peak power values ratio is equal to their avalanche photodiode spectral sensitivity values from [10] reverse ratio. The studied molecules concentration range is $10^{12} \ldots 10^{16} \mathrm{~cm}^{-3}$ and the ranging distance was chosen from 1 up to $8 \mathrm{~m}$. The atmospheric extinction coefficient values $k_{A}$ were taken from $[10]$ and for the two laser radiation wavelengths are equal to 0.17 and $0.135 \mathrm{~km}^{-1}$ respectively.

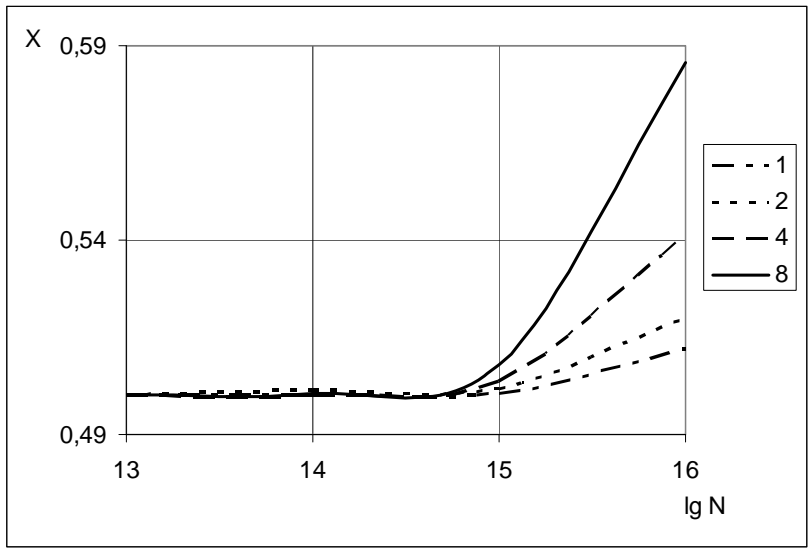

Fig.3. Plot of the DA coefficient $\mathrm{X}$ values vs. iodine molecules concentration $N$ in $10^{15} \mathrm{~cm}^{-3}$ at the distance range $1 \ldots 8 \mathrm{~m}$ (pointed as 1, 2, 4 and 8 respectively).

DA-lidar signal powers ratio simulations by equation (3) were fulfilled using the above described parameters as a function of $\mathrm{I}_{2}$ molecules concentration in the range from $10^{12}$ up to $10^{16} \mathrm{~cm}^{-3}$ and ranging distances from 1 to $8 \mathrm{~m}$. These simulation results are exhibited in Fig.3. As results from Fig.3, the iodine molecules concentration in the range of $10^{12} \ldots 10^{16} \mathrm{~cm}^{-3}$ can be sensed in all of the distance range, but concentration level of $10^{16} \mathrm{~cm}^{-3}$ at $8 \mathrm{~m}$ was exponential due to the high value of $\sigma_{0} N$ as in the usual absorption spectroscopy [3, 8]. For further study would be of interest to compare the results of laser radiation power with the signal weakening as the radiation passes through the measuring volume and it does not overlap the signal recorded by DA photodetector in all of the distance ranges and molecules concentrations with exception of concentration level at $N_{a}=10^{16} \mathrm{~cm}^{-3}$. All of the simulations were fulfilled for laser radiation peak power of $10 \mathrm{~kW}$. The laser radiation peak power value increasing up to $100 \mathrm{~kW}$ creates the sensing efficiency proportionally increased ratio. The $X$ values differences in Fig.2 and Fig.3 are from our experimental accuracy.

\section{CONCLUSION}

Laboratory lidar studies and lidar equation simulation allowed us to get the iodine molecules absorption cross section at $532 \mathrm{~nm}$ wavelength $\sigma_{0}=(1.88 \pm 0.37) 10^{-18} \mathrm{~cm}^{2}$ in iodine molecules concentration range from $7.4210^{15} \mathrm{~cm}^{-3}$ to $32.410^{15} \mathrm{~cm}^{-3}$ in satisfactory confidence with data [3] - 4.6 $10^{-18} \mathrm{~cm}^{2}$ at $590 \mathrm{~nm}$ wavelength.

The iodine molecules absorption cross section at $532 \mathrm{~nm}$ wavelength was taken as the basis of the DA-lidar equation computer simulation. These simulation results corresponded with our DA-lidar experimental data.

\section{REFERENCES}

[1] Privalov, V.E., Shemanin, V.G. (1999). Parameters of differential absorption lidar for detection of molecular iodine in atmosphere. Rus. J. Optical Tech. 66 (2), 40-42.

[2] Privalov, V.E., Shemanin, V.G., Voronina, E.I. (2002). Lidar measurements of iodine molecules concentration. In Proceedings of the SPIE. Vol. 4900, 78-82.

[3] Measures, R.M. (1987). Laser Remote Sensing. Moscow: Mir.

[4] Vorob'eva, L.P., Evtushenko, G.S., Klimkin, V.M. et al. (1995). Cu-laser in problem of iodine radionuclides monitoring. Rus. Atmosphere and Ocean Optics, 8, 1648-1651.

[5] Privalov, V.E., Shemanin, V.G. (1998). Lidars for control and measurements. In Proceedings of the SPIE. Vol. 3345, 6-10.

[6] Privalov, V.E., Shemanin, V.G. (2000). Molecular iodine laser monitoring in the atmosphere. In Proceedings of the SPIE. Vol. 4316, 36-42.

[7] Privalov, V.E., Shemanin, V.G., Voronina, E.I. (2001). Air quality controlling system for industrial region. In Proceedings of the SPIE. Vol. 4680, 112-128.

[8] Zuyev, V.V., Kataev, M.Yu., Makogon, M.M., Mitzel, A.A. (1995). Differential absorption lidar method. Studies real status. Rus. Atmosphere and Ocean Optics, 8, 1136-1164.

[9] Mironov, A.V., Privalov, V.E., Savel'ev, S.K. (1996). Iodine-127 absorption lines calculation according to cuprum vapor laser radiation lines. Rus. Optics and Spectroscopy, 80, 348-350.

[10] Prokhorov, A. M. (Ed.) (1978). Laser Handbook. Vol. II. Moscow: Sov. Radio. 